\title{
Sequential treatment of tyrosine kinase inhibitor and platinum-based doublet chemotherapy on EGFR mutant non-small cell lung cancer: a meta- analysis of randomized controlled clinical trials
}

\author{
This article was published in the following Dove Press journal: \\ OncoTargets and Therapy \\ 28 February 2017 \\ Number of times this article has been viewed
}

\author{
Lifen Qiao',2,* \\ Jin Wang ${ }^{2,3, *}$ \\ Guoxian Long',4 \\ Yueqiang Jiang 1,5 \\ 'Department of Geriatrics, Tongji \\ Hospital, Tongji Medical College, \\ Huazhong University of Science and \\ Technology, Wuhan, China; ${ }^{2}$ Cancer \\ Center, Medical College of Wisconsin, \\ Milwaukee, WI, USA; ${ }^{3}$ Department \\ of Emergency, ${ }^{4}$ Department of \\ Oncology, Tongji Hospital, Tongji \\ Medical College, Huazhong University \\ of Science and Technology, Wuhan, \\ China; ${ }^{5}$ Department of Toxicology \\ and Pharmacology, Medical College \\ of Wisconsin, Milwaukee, WI, USA \\ *These authors contributed equally \\ to this work
}

\begin{abstract}
There is debate surrounding which treatment is superior in overall survival (OS) rates in patients with epidermal growth factor receptor (EGFR) mutant non-small cell lung cancer (NSCLC); first-line tyrosine kinase inhibitor (TKI) followed by second-line platinum-based doublet chemotherapy (PCT), or the reverse sequence. Cross treatment of first- and second-line TKI and PCT makes it difficult to deduce which sequence (TKI-PCT or PCT-TKI) is better for OS. Using the keywords "lung cancer" and "EGFR" we identified clinical trials within the PubMed database which were published between January 2006 and November 2016. Basic characteristics and OS with hazard ratio and 95\% confidence intervals were searched and analyzed. In total, 457 articles were reviewed and nine clinical trials with 1,876 patients were of sufficient quality for further analysis. Fixed effects models were performed to pool the data in this meta-analysis. All nine studies were open-labeled, multicenter, Phase III randomized controlled clinical trials. The pooled hazard ratio was 0.96 (95\% confidence interval: $0.84-1.10$ ) for OS between first-line TKI followed by second-line PCT compared to the reverse sequence. No statistically significant heterogeneity $\left(I^{2}=0, P=0.553\right)$ nor publication bias (Egger's $P=0.991$ ) was observed among these studies. In conclusion, there was no OS benefit between first-line TKI followed by second-line PCT compared to the reverse sequence in EGFR mutant NSCLC patients. Chemotherapy was still useful and irreplaceable for the treatment of NSCLC, especially for those patients with EGFR unavailable for testing.
\end{abstract}

Keywords: TKI, EGFR, chemotherapy, lung cancer, meta-analysis

\section{Introduction}

Non-small cell lung cancer (NSCLC) accounts for $75 \%-80 \%$ of all lung cancer, which remains a leading cause of death all over the world. In China, it is estimated that more than 733,000 new cases of lung cancer will be diagnosed in 2015 and as many as 610,200 deaths will occur due to the fact that most patients will be diagnosed at late stage. This corresponds to 1,672 Chinese dying of lung cancer per day on average. ${ }^{1}$

Platinum-based doublet chemotherapy (PCT) is currently used as the main treatment for IIIB/IV stage NSCLC. Tyrosine kinase inhibitor (TKI), which includes gefitinib, erlotinib, and afatinib, is also recommended as first-line treatment for NSCLC and has been widely used on epidermal growth factor receptor (EGFR) mutant NSCLC, showing a favorable response, better progression free survival (PFS), and less side effects than conventional PCT. ${ }^{2}$ Icotinib, a TKI originating from China, with less side effects than gefitinib has been proved not inferior to gefitinib as a second-line
Correspondence: Yueqiang Jiang Department of Geriatrics, Tongji Hospital, Tongji Medical College, Huazhong University of Science and Technology, Jiefang Road No 1095, Wuhan 430030, China

Tel +862783663010

Email jyqwh@163.com 
treatment, was approved and recommended by the Chinese government in $2011 .^{3}$

It is more common to find the EGFR mutation in Chinese and other Asian NSCLC patients than in patients from Western countries, consequently explaining why most clinical trials have taken place in Asian regions. ${ }^{4}$ Furthermore, under regular treatment, patients with the EGFR mutation have a significantly longer survival time than wild types, meaning EGFR mutant patients have more opportunity for further treatment or to partake in clinical trials than wild types, which might further prolong their life span.,

In these EGFR mutant NSCLC patients, PFS has been greatly prolonged with the first-line treatment of TKI rather than with conventional PCT. However, overall survival (OS) has not increased. ${ }^{5-14}$ It is suggested that this is because of the cross treatment of TKI and chemotherapy, especially from PCT, during the first- and second-line treatments. As a result, this implies a very interesting question on whether there is the same OS benefit by treating patients with a first-line of TKI followed by second-line PCT (TKI-PCT arm) compared to the reverse sequence (PCT-TKI arm) on late stage EGFR mutant NSCLC patients. In this study, we conducted a meta-analysis to evaluate and further understand and discuss the efficiency of treating patients with first-line TKI followed by second-line conventional PCT compared to first-line PCT followed by second-line TKI on EGFR mutant NSCLC patients.

\section{Methods}

\section{Literature search strategy}

We searched in PubMed using the keywords "lung cancer" and "EGFR" for papers published between January 1, 2006 and November 1, 2016, and restricted the literature type to "clinical trial". The language was limited to English.

\section{Selection criteria}

Studies were included if they met the following criteria: 1) EGFR mutation status reported; 2) used and compared treatment of PCT and TKI on EGFR mutant NSCLC patients; 3) OS including hazard ratio (HR) with 95\% confidence interval (CI) available to analyze. Zhang et al's study analyzed the sequential treatment of TKI and chemotherapy using poor and limited data because many studies they cited did not update their follow up data at that time. ${ }^{15}$ In our analysis, clinical trials that had renewed information, had been updated or were final reports were also included.

\section{Data extraction}

Two investigators (L Qiao and J Wang) performed the literature search, screening, and data extraction independently and discussed with a third investigator ( $\mathrm{Y}$ Jiang) if they could not reach a consensus. The following variables were extracted from the included studies: first author, race, latest year published, chemotherapy regimen, TKI regimen, and number of patients.

\section{Statistics analysis}

In this meta-analysis, Stata (version 12.0, Stata Corporation, College Station, TX, USA) was used to analyze our data. OS was assessed with HR and its 95\% CI. I-square test $\left(I^{2}\right)$ was performed to assess the heterogeneity among included trials. Begg's and Egger's tests were conducted to evaluate publication bias.

\section{Results}

\section{Literature research and basic characteristics of studies included}

A total of 457 articles were located in PubMed using the keywords and limitations provided previously in the "Literature search strategy" section. In total, nine clinical trials were included in our analysis. The detailed process of screening is presented in Figure 1. The characteristics of chosen articles are shown in Table 1. Some studies had both the OS data published from the original paper available and long-term follow-up data presented in a paper at a later date, which caused data to vary. For example, the medium OS in Mitsudomi et al's clinical trial reports were renewed between the first edition in 2010 to the latest in $2012 .^{8,16}$

In early clinical trials, the precise mechanism of how TKI worked on NSCLC was unknown, so the studied population did not focus on EGFR mutant patients. ${ }^{5,6}$ However, after subgroup analysis confirmed that EGFR mutation status played an essential role, studies then focused on EGFR mutant NSCLC patients.

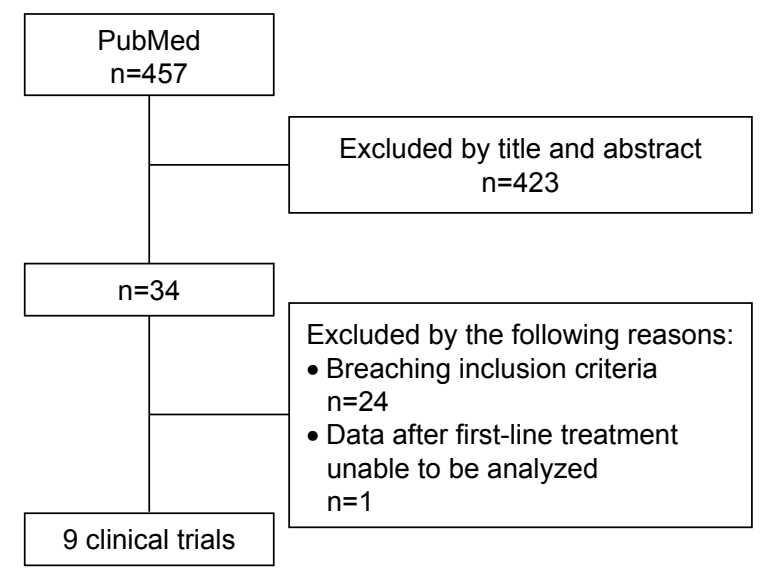

Figure I Flowchart of publication selection process. 
Table I Characteristics of the nine included clinical trials

\begin{tabular}{|c|c|c|c|c|c|c|}
\hline \multirow[t]{2}{*}{ Study } & \multirow{2}{*}{$\begin{array}{l}\text { Year } \\
\text { published }\end{array}$} & \multicolumn{2}{|l|}{ EGFR $^{+}$} & \multicolumn{2}{|l|}{ Regimen } & \multirow{2}{*}{$\begin{array}{l}\text { Medium OS } \\
\text { (month) }\end{array}$} \\
\hline & & Race & $\begin{array}{l}\text { Number } \\
\text { of patients }\end{array}$ & First-line & $\overline{\text { Second-line }}$ & \\
\hline Inoue et $a ;_{; 3}^{13}$ & $2013 ; 2015$ & Asian & 114 & Taxol $+\mathrm{P}$ & Gefitinib & 27.6 \\
\hline Miyauchi et al ${ }^{18}$ & & & 114 & Gefitinib & PCT & 28.9 \\
\hline \multirow[t]{2}{*}{ Zhou et al ${ }^{14}$} & 2015 & Asian & 72 & $\mathrm{Gem}+\mathrm{P}$ & TKI & 32 \\
\hline & & & 82 & Erlotinib & PCT & 28 \\
\hline \multirow[t]{2}{*}{ Wu et $\mathrm{al}^{7}$} & 2015 & Asian & 107 & Gem $+P$ & Erlotinib & 24.7 \\
\hline & & & 110 & Erlotinib & Gem $+P$ & 29.4 \\
\hline Wu et al; $;$ & $2014 ; 2015$ & Asian & 108 & Gem $+P$ & TKI & 23.5 \\
\hline Yang et al"I & & & 216 & Afatinib & PCT & 23.1 \\
\hline Rosell et al; ${ }^{12}$ & $2012 ; 2014$ & Caucasian and Hispanic & 88 & PCT & TKI & 16.5 \\
\hline Leon et $\mathrm{al}^{17}$ & & & 86 & Erlotinib & PCT & 22.9 \\
\hline Sequist et al; ${ }^{10}$ & $2013 ; 2015$ & $M i x^{a}$ & 104 & $\mathrm{Pem}+\mathrm{P}$ & TKI & 28.2 \\
\hline Yang et al" & & & 203 & Afatinib & PCT & 28.2 \\
\hline Mitsudomi et al; ${ }^{8}$ & $2010 ; 2012$ & Asian & 86 & $\mathrm{Doc}+\mathrm{P}$ & Gefitinib & 38.8 \\
\hline Mitsudomi et al ${ }^{16}$ & & & 86 & Gefitinib & PCT & 35.5 \\
\hline \multirow[t]{2}{*}{ Gridelli et al ${ }^{6}$} & 2012 & Caucasian & 20 & $\mathrm{Gem}+\mathrm{P}$ & Erlotinib & 32.5 \\
\hline & & & 19 & Erlotinib & $\mathrm{Gem}+\mathrm{P}$ & 18.1 \\
\hline \multirow[t]{2}{*}{ Fukuoka et al ${ }^{5}$} & 2011 & Asian & 129 & Taxol + P & TKI & 21.9 \\
\hline & & & 132 & Gefitinib & PCT & 21.2 \\
\hline
\end{tabular}

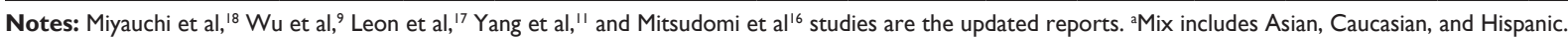

Abbreviations: Doc, docetaxel; Gem, gemcitabine; OS, overall survival; P, platinum; PCT, platinum-based doublet chemotherapy; Pem, pemetrexed; TKI, tyrosine kinase inhibitor.

All of the nine studies we analyzed were open-labeled, multicenter, Phase III randomized controlled clinical trials (RCTs). A total of 1,876 patients were included in our analysis from these nine studies, with 1,048 patients in the TKI-PCT arm and 828 patients in the PCT-TKI arm. The first-line TKI included only gefitinib, erlotinib, and afatinib as icotinib had not yet been approved in China during the time of these studies, while the second- or later-line included all four types of TKIs. Although there is a higher incidence of EGFR mutation found in Asians, three studies containing Caucasian and Hispanic races were also included. ${ }^{6,10,12}$ Sequist et al's study contained all three races above. ${ }^{10}$

\section{Overall survival}

In all nine studies, using fixed effects models to pool the data, results revealed that there was no OS benefit (HR: 0.96, 95\% CI: 0.84-1.10) in the TKI-PCT arm compared to the PCT-TKI arm (Figure 2). The $I^{2}$ test $\left(I^{2}=0 \%, P=0.553\right)$ showed no statistically significant heterogeneity among these studies (Figure 2). The Egger's test had a $P$-value of 0.991, suggesting that there was no statistical publication bias for these two treatment sequences (Figure 3).

Gridelli et al's study, which was performed on a Caucasian population, indicated a tendency for a worse medium OS in the TKI-PCT arm than in the PCT-TKI arm (18.1 months vs 32.5 months, HR: $1.58,95 \%$ CI: $0.70-3.57) .{ }^{6}$ However, only a small population size was included in their study, which might weaken their interpretation of the result. Leon et al's updated study, using an approach to account for treatment crossover, seemed to indicate a tendency for a better OS in the TKI-PCT arm than in the reverse sequence (22.9 months vs 16.5 months, HR: $0.70,95 \%$ CI: $0.27-1.78) .{ }^{17}$ Miyauchi et al's updated study indicated a similar OS benefit in both arms (28.9 months vs 27.6 months, HR: 0.77, 95\% CI: $0.52-1.14) .{ }^{18}$ Other six clinical trials also showed detailed information of medium OS with HR and CI and Kaplan-Meier curve for each group of the EGFR mutant population.

Analyzing the OS, results indicated that the TKI-PCT arm was not superior to the PCT-TKI arm for advanced stage EGFR mutant NSCLC patients.

\section{Discussion}

TKI has been a milestone in the treatment of EGFR mutant NSCLC when prescribed as first-line, greatly improving PFS as well as reducing side effects that patients treated with conventional PCT encounter. It is necessary to test for EGFR mutation status before the treatment of NSCLC if possible, using detection methods such as cytology sample and liquid biopsy. From first generation TKIs gefitinib, erlotinib, and icotinib, to second generation TKI afatinib, more and more effective TKIs are being produced, promoted by the research of targeted therapy which now remains a hotspot in cancer research. The first generation TKI is a reversible antagonist 


\begin{tabular}{|c|c|c|}
\hline Study ID & $\mathrm{HR}(95 \% \mathrm{Cl})$ & $\%$ weight \\
\hline Fukuoka et al ${ }^{5}$ & $1.00(0.76-1.33)$ & 21.96 \\
\hline Mitsudomi et $\mathrm{al}^{16}$ & $1.19(0.77-1.83)$ & 9.11 \\
\hline Gridelli et $\mathrm{al}^{6}$ & $1.58(0.70-3.57)$ & 2.59 \\
\hline Rosell et al ${ }^{12}$ & $0.70(0.27-1.78)$ & 1.93 \\
\hline Sequist et al ${ }^{10}$ & $0.88(0.66-1.77)$ & 7.07 \\
\hline Wu et $\mathrm{al}^{9}$ & $0.93(0.72-1.22)$ & 24.73 \\
\hline Miyauchi et al ${ }^{18}$ & $0.77(0.52-1.14)$ & 11.16 \\
\hline Wu et $\mathrm{al}^{7}$ & $0.74(0.47-1.17)$ & 8.27 \\
\hline Zhou et al ${ }^{14}$ & $1.19(0.83-1.71)$ & 13.17 \\
\hline Overall $\left(I^{2}=0.0 \%, P=0.553\right)$ & $0.96(0.84-1.10)$ & 100 \\
\hline 0.27 & 3.7 & \\
\hline
\end{tabular}

Figure 2 Forest plot of OS comparing TKI-PCT arm and PCT-TKI arm.

Notes: TKI-PCT arm: patients treated with first-line TKI followed by second-line PCT; PCT-TKI arm: patients treated with first-line PCT followed by second-line TKI. Abbreviations: $\mathrm{Cl}$, confidence interval; HR, hazard ratio; OS, overall survival; PCT, platinum-based doublet chemotherapy; TKI, tyrosine kinase inhibitor.

of EGFR, while the second generation irreversible. However, no clinical trial has yet indicated that second generation TKI improves PFS or OS significantly more than first generation TKIs. As all of them are recommended as first-line treatment to EGFR mutant NSCLC, our analysis focused on studies using either gefitinib, erlotinib, afatinib or combined. However, icotinib, proven not inferior to gefitinib by a phase III RCT in 2013, was only used as a second- or laterline in the studies chosen in our paper. ${ }^{3}$ In patients prescribed with TKIs, almost all of them developed drug resistance over time. There are multiple reasons for this with the most important being that $\sim 60 \%$ of patients have been found to have a second mutation called T790M in exon 20 of EGFR. ${ }^{19}$

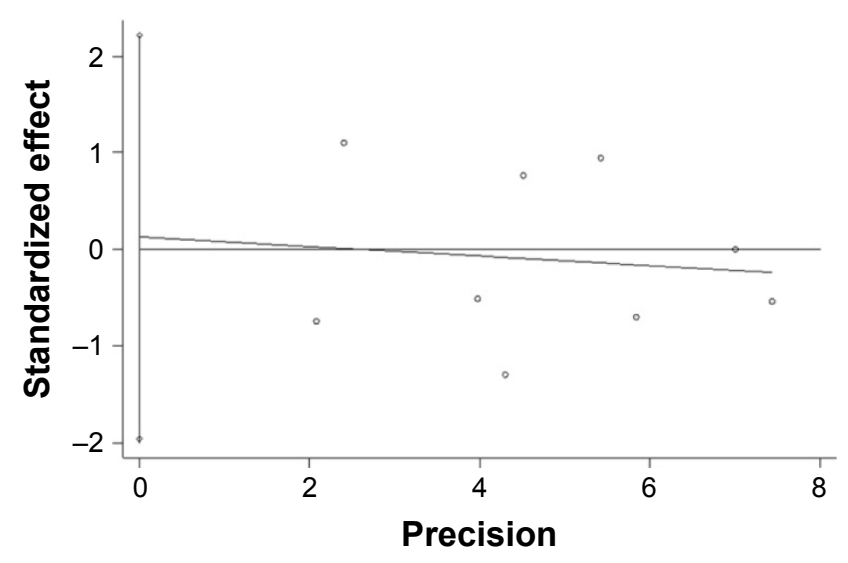

Figure 3 Egger's publication bias plot.
The third generation TKI, AZD9291, which was approved by the US FDA in 2015, is able to fight against the T790M mutation and stay effective after progression of first-line TKI. However, AZD9291's efficiency could be reduced if met or combined with different mutations, such as C797S. ${ }^{20,21}$ It is considered that chemotherapy, especially PCT, is an effective method in the treatment of EGFR mutant NSCLC, however, no clinical trials had been performed until now to directly compare AZD9291 and PCT after progression of first-line TKI. After progression of first-line TKI, some patients continue with TKIs without changing their therapeutic strategy, while some change to PCT while still continuing with TKIs. This combined approach is thought to get rid of the "flash effect" and the combination of the two treatment methods is thought to be better than the single therapy. However, it has been recently reported in a Phase III RCT that continuation of TKI did not prolong PFS when receiving PCT for EGFR mutant NSCLC patients after the progression of first-line TKI. ${ }^{22}$ It is implied that in these patients, second-line PCT, which remains a better option than continuing TKI without changing, is effective and irreplaceable. Although targeted and personalized therapy is becoming more and more important, it seems from our analysis that PCT was more beneficial than we thought, and sequential PCT and TKI could offer a better prognosis than a single treatment method, no matter which sequence it was delivered. However, because PFS and quality of life is reported to be better when first-line 
treatment is with TKI than with PCT, care should be taken in interpreting the results of our analysis.

Two main EGFR mutation types include exon 19 deletion and 21-point mutation L858R. Although TKI has been shown in RCTs to improve PFS, no OS improvement has been reported, except for afatinib in a subgroup of exon 19 deletion. ${ }^{11}$ However, $74 \%$ of this subgroup received subsequent systemic therapy, that is to say, most of them received PCT apparently higher than that of other subgroup (for example, the L858R subgroup 66\%), which might play an essential role in OS improvement. However, in our study we found it hard analyze this subgroup of OS benefit because many studies did not present this data.

There are some limitations in our paper. First, different cycles of chemotherapy were performed in the different studies we analyzed, which might result in the bias of the analysis. For example, in Wu et al's ${ }^{7}$ study only four cycles of PCT were performed in the PCT-TKI arm, compared to the fact that three to six cycles of chemotherapy were performed in Mitsudomi et al's ${ }^{16}$ study. What's more, for the PCT-TKI arm in many of the clinical trials, after the prescribed duration of chemotherapy, no matter whether there was progression or not, patients would then undertake TKI, which would restrict and lessen the function of chemotherapy. However, in the TKI-PCT arm, as a salvable therapy after the progression of TKIs, PCT was always given as often as required and accepted by patients.

Second, the difference of chemotherapy regimens could cause bias. Due to the lack of head to head comparison, PCT regimens including gemcitabine, taxol, or pemetrexed are all considered to have similar therapeutic effects on NSCLC. Meanwhile, pemetrexed has less side effects than other TKIs and a RCT has also proven it to be effective as maintenance therapy after first-line PCT in non-squamous NSCLC. ${ }^{23}$ Also, as a second-line, pemetrexed had significantly better clinical efficacy in patients with susceptible EGFR mutations after progression of first-line TKI. ${ }^{24}$ However, except for Sequist et al's study, the other eight studies did not use pemetrexed in first-line treatment in order for us to compare TKIs in our analysis, due to the fact that pemetrexed was approved as first-line treatment by FDA in 2009. ${ }^{10}$

Finally, although using high valuated data, some patients did not follow cross treatment, and this seemed to result in some bias. However, it might not affect the final analysis. For one reason, the crossed population took a major part of the EGFR positive patients, which contributed to most of the survival time, and for another reason, the population with cross treatment of TKI and PCT had a better life span than patients without crossing. Except for Gridelli et al's study which was originally designed to compare TKI-PCT with PCT-TKI in unselected NSCLC patients, the other eight trials were designed to compare first-line treatment of TKI with PCT. ${ }^{6}$

In our analysis, results indicate that for EGFR mutant NSCLC patients, first-line TKI followed by secondline PCT is not superior to first-line PCT followed by second-line TKI. Chemotherapy still remains an important treatment option also. ${ }^{25}$ Further prospective clinical trials are required to continue to study this question. We are now emphasizing more and more on personalized and targeted therapy, however, chemotherapy is still useful and should still be considered for patients after the progression of TKI and especially in patients with an undiagnosed mutation status.

\section{Disclosure}

The authors report no conflicts of interest in this work.

\section{References}

1. Chen W, Zheng R, Baade PD, et al. Cancer statistics in China, 2015. CA Cancer J Clin. 2016;66(2):115-132.

2. Ettinger DS, Wood DE, Akerley W, et al. Non-small cell lung cancer, version 6. 2015. J Natl Compr Canc Netw. 2015;13(5):515-524.

3. Shi Y, Zhang L, Liu X, et al. Icotinib versus gefitinib in previously treated advanced non-small-cell lung cancer (ICOGEN): a randomised, double-blind phase 3 non-inferiority trial. Lancet Oncol. 2013;14(10): 953-961.

4. Rosell R, Moran T, Queralt C, et al. Screening for epidermal growth factor receptor mutations in lung cancer. N Engl J Med. 2009;361(10): 958-967.

5. Fukuoka M, Wu YL, Thongprasert S, et al. Biomarker analyses and final overall survival results from a phase III, randomized, open-label, first-line study of gefitinib versus carboplatin/paclitaxel in clinically selected patients with advanced non-small-cell lung cancer in Asia (IPASS). J Clin Oncol. 2011;29(21):2866-2874.

6. Gridelli C, Ciardiello F, Gallo C, et al. First-line erlotinib followed by second-line cisplatin-gemcitabine chemotherapy in advanced nonsmall-cell lung cancer: the TORCH randomized trial. J Clin Oncol. 2012;30(24):3002-3011.

7. Wu YL, Zhou C, Liam CK, et al. First-line erlotinib versus gemcitabine/ cisplatin in patients with advanced EGFR mutation-positive non-smallcell lung cancer: analyses from the phase III, randomized, open-label, ENSURE study. Ann Oncol. 2015;26(9):1883-1889.

8. Mitsudomi T, Morita S, Yatabe Y, et al. Gefitinib versus cisplatin plus docetaxel in patients with non-small-cell lung cancer harbouring mutations of the epidermal growth factor receptor (WJTOG3405): an open label, randomised phase 3 trial. Lancet Oncol. 2010;11(2): $121-128$.

9. Wu YL, Zhou C, Hu CP, et al. Afatinib versus cisplatin plus gemcitabine for first-line treatment of Asian patients with advanced non-small-cell lung cancer harbouring EGFR mutations (LUX-Lung 6): an open-label, randomised phase 3 trial. Lancet Oncol. 2014;15(2):213-222.

10. Sequist LV, Yang JC, Yamamoto N, et al. Phase III study of afatinib or cisplatin plus pemetrexed in patients with metastatic lung adenocarcinoma with EGFR mutations. J Clin Oncol. 2013;31(27): 3327-3334.

11. Yang JC, Wu YL, Schuler M, et al. Afatinib versus cisplatin-based chemotherapy for EGFR mutation-positive lung adenocarcinoma (LUXLung 3 and LUX-Lung 6): analysis of overall survival data from two randomised, phase 3 trials. Lancet Oncol. 2015;16(2):141-151. 
12. Rosell R, Carcereny E, Gervais R, et al. Erlotinib versus standard chemotherapy as first-line treatment for European patients with advanced EGFR mutation-positive non-small-cell lung cancer (EURTAC): a multicentre, open-label, randomised phase 3 trial. Lancet Oncol. 2012; 13(3):239-246.

13. Inoue A, Kobayashi K, Maemondo M, et al. Updated overall survival results from a randomized phase III trial comparing gefitinib with carboplatinpaclitaxel for chemo-naive non-small cell lung cancer with sensitive EGFR gene mutations (NEJ002). Ann Oncol. 2013;24(1):54-59.

14. Zhou $\mathrm{C}, \mathrm{Wu} \mathrm{YL}$, Chen $\mathrm{G}$, et al. Final overall survival results from a randomised, phase III study of erlotinib versus chemotherapy as first-line treatment of EGFR mutation-positive advanced non-small-cell lung cancer (OPTIMAL, CTONG-0802). Ann Oncol. 2015;26(9):1877-1883.

15. Zhang Y, Sun Y, Wang L, et al. Sequential treatment of tyrosine kinase inhibitors and chemotherapy for EGFR-mutated non-small cell lung cancer: a meta-analysis of Phase III trials. Onco Targets Ther. 2013;6: 1771-1777.

16. Mitsudomi T, Morita S, Yatabe Y, et al. Updated overall survival results of WJTOG 3405, a randomized phase III trial comparing gefitinib $(\mathrm{G})$ with cisplatin plus docetaxel $(\mathrm{CD})$ as the first-line treatment for patients with non-small cell lung cancer harboring mutations of the epidermal growth factor receptor (EGFR). J Clin Oncol. 2012; 30(suppl; abstr 7521)

17. Leon LF, Golsorkhi A, Liu S, Drozdowskyj A, Rosell R. Overall survival analyses of first-line erlotinib versus chemotherapy in the EURTAC study population controlling for the use of post-study therapy. Ann Oncol. 2014;25(suppl 4):iv426-iv470.

18. Miyauchi E, Inoue A, Kobayashi K, et al. Efficacy of chemotherapy after first-line gefitinib therapy in EGFR mutation-positive advanced non-small cell lung cancer-data from a randomized Phase III study comparing gefitinib with carboplatin plus paclitaxel (NEJ002). Jpn J Clin Oncol. 2015;45(7):670-676.
19. Yu HA, Arcila ME, Rekhtman N, et al. Analysis of tumor specimens at the time of acquired resistance to EGFR-TKI therapy in 155 patients with EGFR-mutant lung cancers. Clin Cancer Res. 2013;19(8): 2240-2247.

20. Jänne PA, Yang JC, Kim DW, et al. AZD9291 in EGFR inhibitor-resistant non-small-cell lung cancer. N Eng J Med. 2015;372(18):1689-1699.

21. Wang S, Tsui ST, Liu C, Song Y, Liu D. EGFR C797S mutation mediates resistance to third-generation inhibitors in T790M-positive non-small cell lung cancer. J Hematol Oncol. 2016;9(1):59.

22. Soria JC, Wu YL, Nakagawa K, et al. Gefitinib plus chemotherapy versus placebo plus chemotherapy in EGFR-mutation-positive non-small-cell lung cancer after progression on first-line gefitinib (IMPRESS): a phase 3 randomised trial. Lancet Oncol. 2015;16(8):990-998.

23. Paz-Ares L, de Marinis F, Dediu M, et al. Maintenance therapy with pemetrexed plus best supportive care versus placebo plus best supportive care after induction therapy with pemetrexed plus cisplatin for advanced non-squamous non-small-cell lung cancer (PARAMOUNT): a double-blind, phase 3, randomised controlled trial. Lancet Oncol. 2012;13(3):247-255.

24. Yang CJ, Tsai MJ, Hung JY, et al. Pemetrexed had significantly better clinical efficacy in patients with stage IV lung adenocarcinoma with susceptible EGFR mutations receiving platinum-based chemotherapy after developing resistance to the first-line gefitinib treatment. Onco Targets Ther. 2016;9:1579-1587.

25. Kiura K. Chemotherapy for lung cancer: still alive! Jpn J Clin Oncol. 2015;45(7):609-610
OncoTargets and Therapy

\section{Publish your work in this journal}

OncoTargets and Therapy is an international, peer-reviewed, open access journal focusing on the pathological basis of all cancers, potential targets for therapy and treatment protocols employed to improve the management of cancer patients. The journal also focuses on the impact of management programs and new therapeutic agents and protocols on

\section{Dovepress}

patient perspectives such as quality of life, adherence and satisfaction The manuscript management system is completely online and includes a very quick and fair peer-review system, which is all easy to use. Visit http://www.dovepress.com/testimonials.php to read real quotes from published authors. 